\title{
On the temperature dependence of electronically non-adiabatic vibrational energy transfer in molecule-surface collisions
}

\author{
Daniel Matsiev, ${ }^{a}$ Zhisheng $\mathrm{Li}^{a}{ }^{a}$ Russell Cooper, ${ }^{a b}$ Igor Rahinov, ${ }^{a c}$ \\ Christof Bartels, ${ }^{a b}$ Daniel J. Auerbach $^{a d}$ and Alec M. Wodtke ${ }^{* a b e}$
}

\author{
Received 4th August 2010, Accepted 1st October 2010 \\ DOI: $10.1039 /$ c0cp01418d
}

\begin{abstract}
Here we extend a recently introduced state-to-state kinetic model describing single- and multi-quantum vibrational excitation of molecular beams of NO scattering from a $\mathrm{Au}(111)$ metal surface. We derive an analytical expression for the rate of electronically non-adiabatic vibrational energy transfer, which is then employed in the analysis of the temperature dependence of the kinetics of direct overtone and two-step sequential energy transfer mechanisms. We show that the Arrhenius surface temperature dependence for vibrational excitation probability reported in many previous studies emerges as a low temperature limit of a more general solution that describes the approach to thermal equilibrium in the limit of infinite interaction time and that the pre-exponential term of the Arrhenius expression can be used not only to distinguish between the direct overtone and sequential mechanisms, but also to deduce their relative contributions. We also apply the analytical expression for the vibrational energy transfer rates introduced in this work to the full kinetic model and obtain an excellent fit to experimental data, the results of which show how to extract numerical values of the molecule-surface coupling strength and its fundamental properties.
\end{abstract}

\section{Introduction}

Chemical reactivity at metal surfaces plays an important role in the modern industrial world. Understanding of fundamental energy transfer processes between molecular and solid surface degrees of freedom contributes to our ability to develop new materials, catalysts, and synthetic routes. Among all possible pathways for energy exchange, coupling of the nuclear vibrational motion to electronic excitations in metals is still one of the least understood and is in fact often neglected. Since the pioneering work of Rettner et al., ${ }^{1}$ where direct singlequantum vibrational excitation of $\mathrm{NO}$ was detected in collisions with $\operatorname{Ag}(111)$, such electronically non-adiabatic vibrational energy transfer at metal surfaces has been observed experimentally in several systems..$^{2-4}$ Electronically mediated singlequantum vibrational energy transfer (and, more generally, the underlying molecule-surface charge transfer) was also a subject of significant theoretical effort. ${ }^{5-16}$

In a recent paper ${ }^{17}$ we reported observations of electronically non-adiabatic multi-quantum vibrational excitation in NO collisions with $\mathrm{Au}(111)$, where absolute vibrational excitation probabilities of $\mathrm{NO}(v=0 \rightarrow 1,2)$ were measured as a function of surface temperature, and a state-to-state kinetic

${ }^{a}$ Department of Chemistry and Biochemistry,

University of California Santa Barbara, Santa Barbara,

CA 93106-9510,USA.E-mail: alec.wodtke@mpibpc.mpg.de

${ }^{b}$ Institut für Physikalische Chemie, Georg-August-Universität,

Tammannstraße 6, 37077 Göttingen, Germany

${ }^{c}$ Department of Natural Sciences, The Open University of Israel,

108 Ravutski Street, Raanana 43107, Israel

${ }^{d}$ GRT Inc., 861 Ward Drive, Santa Barbara, CA 93111, USA

${ }^{e}$ Max-Planck-Institut Für Biophysikalische Chemie,

Karl-Friedrich-Bonhoeffer-Institut, Am Fassberg 11,

37077 Göttingen, Germany model was introduced to help interpret the experimental data. That model assumed the rates of the energy transfer $k_{v v^{\prime}}$ to be proportional to the number of excited electron-hole pairs whose excitation energy matches the vibrational spacing of the molecule, leading to expressions of the following type:

$$
k_{v v^{\prime}}=\xi_{v v^{\prime}} \int \rho(\varepsilon) f\left(T_{\mathrm{S}}, \varepsilon\right) \rho\left(\varepsilon-E_{v v^{\prime}}\right)\left[1-f\left(T_{\mathrm{S}}, \varepsilon-E_{v v^{\prime}}\right)\right] \mathrm{d} \varepsilon
$$

where $v$ and $v^{\prime}$ are the vibrational quantum numbers of the initial and final vibrational states, $E_{v v^{\prime}}=\hbar \omega_{0} \times\left(v^{\prime}-v\right)$ is the transferred vibrational energy, $\rho(\varepsilon)$ is the density of electronic states, $f\left(T_{\mathrm{S}}, \varepsilon\right)$ is the Fermi-Dirac probability distribution at the surface temperature $T_{\mathrm{S}}$, and $\xi_{v v^{\prime}}$ is a temperature independent coefficient that represents the electronically non-adiabatic interactions between the gas molecule and the metal surface. The model further assumes that the population of vibrationally excited $\operatorname{NO}(v=1,2)$ is determined by a dynamic approach to equilibrium with the surface controlled by rates of excitation given by (1), leading to a system of linear rate equations

$$
\frac{\mathrm{d} n_{v^{\prime}}\left(t, T_{\mathrm{S}}\right)}{\mathrm{d} t}=\sum k_{v v^{\prime}} n_{v, v^{\prime}}\left(t, T_{\mathrm{S}}\right)
$$

By adjusting the values of $\xi_{v v^{\prime}}$, the solutions of (2) provided an excellent fit to the experimental data, quantitatively reproducing the observed surface temperature dependence for both $v=0 \rightarrow 1$ and 2 vibrationally inelastic channels. In our previous work the rate constant integrals (1) were evaluated numerically, thus masking in some ways a clear analysis of the temperature dependence.

In this work we derive a fully analytical version of the above described state-to-state model. This allows us to understand 
the emergence of the previously proposed and experimentally observed Arrhenius dependence of the absolute vibrational excitation probability $P$ on surface temperature ${ }^{1,18,19}$

$$
P=A e^{-\frac{\Delta E_{\mathrm{vib}}}{k_{\mathrm{b}} T_{\mathrm{S}}}}
$$

from the kinetic model, examine the Arrhenius pre-exponents as a function of the interaction time and the surface temperature, and to discover the departure from the Arrhenius behavior at high temperatures. This analysis also allows us to determine the relative importance of direct two-quantum and sequential single-quantum excitation pathways, where the latter proceeds via an intermediate $\operatorname{NO}(v=1)$ state to $\operatorname{NO}(v=2)$. Furthermore, this analysis makes it possible to relate the observed absolute vibrational excitation probabilities to the strength of electronically non-adiabatic coupling.

\section{Experimental}

The experimental observations that provide motivation for this work were recently presented in ref. 17. Briefly, pulsed supersonic molecular beams of NO were scattered from a single crystal $\mathrm{Au}(111)$ surface in an ultra-high vacuum apparatus ${ }^{20}$ and quantum state specific REMPI spectroscopy was carried out on both incident and scattered beams. With proper corrections for all relevant experimental differences between scattered and incident beams (such as temporal and angular distributions, Franck-Condon factors, laser power, detector gain, etc.), the ratios of REMPI intensities yield absolute vibrational excitation probabilities, which were measured at several surface temperatures between 300 and $1000 \mathrm{~K}$ at an incidence kinetic energy of $0.9 \mathrm{eV}$.

\section{Comments on ref. 17}

The most fascinating aspect of this experimental work is arguably the observation of concurrent single- and multiquantum vibrational excitation, where absolute excitation probabilities as high as $2.5 \%$ for $\mathrm{NO}(v=0 \rightarrow 1)$ and $0.15 \%$ for $\mathrm{NO}(v=0 \rightarrow 2)$ were measured. Both channels exhibit an Arrhenius dependence on $T_{\mathrm{S}}$, see Fig. 1. Specifically, the data points are confined to a straight line with the slopeeffective Arrhenius activation energy - given by the magnitude of the transferred vibrational energy, $0.236(\Delta v=1)$ and $0.472 \mathrm{eV}(\Delta v=2)$. We may also derive Arrhenius preexponential factors from these experiments, $0.38(\Delta v=1)$ and $0.46(\Delta v=2)$, see Fig. 1. The narrow angular distributions of the scattered vibrationally excited molecules ${ }^{17}$ indicate direct scattering and no trapping-desorption. Collision times under these conditions are expected to be less than a picosecond.

Arrhenius $T_{\mathrm{S}}$ dependence (3) such as shown in Fig. 1 has been previously recognized as a signature of an electronically non-adiabatic energy transfer mediated by free electrons in the metal surface. ${ }^{1,18,19,21}$ However, a complete interpretation of the physical meaning of the Arrhenius parameters derived from experiment has been lacking.

A number of general points are worth mentioning. In this mechanism, the final vibrational energy in the scattered NO

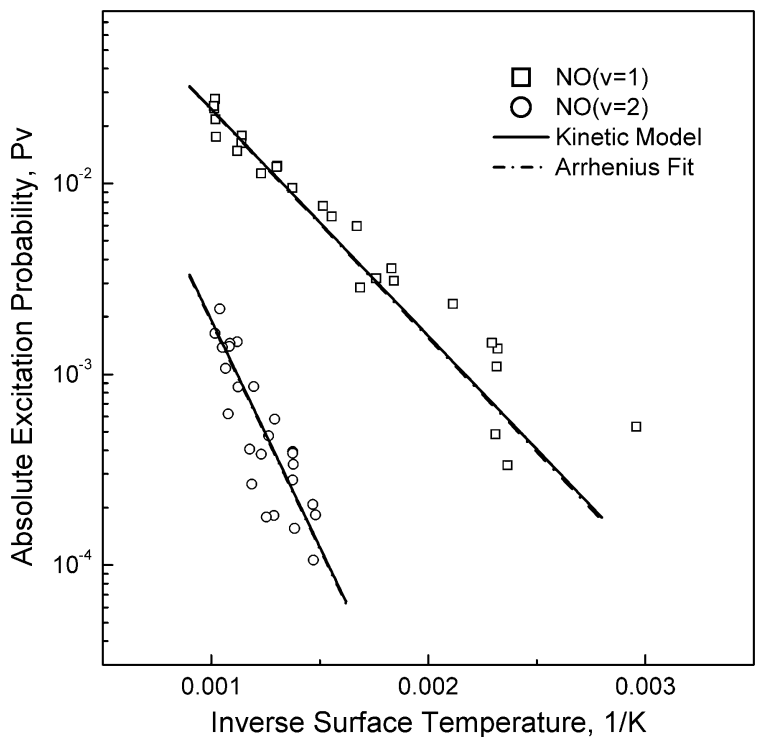

Fig. 1 Arrhenius plot of the absolute vibrational excitation probabilities of $\mathrm{NO}(v=0 \rightarrow 1)$ and $\mathrm{NO}(v=0 \rightarrow 2)$ surface temperature dependence. Scattered symbols denote experimental data; solid lines represent the full kinetic model fit (see section 5.3); dash-dot lines show the canonical Arrhenius fits with $E_{\mathrm{a}}=0.236 \mathrm{eV}, A_{01}=0.38$ and $E_{\mathrm{a}}=0.472 \mathrm{eV}, A_{02}=0.46$ for $\mathrm{NO}(v=1)$ and $(v=2)$ respectively. ${ }^{17}$

molecules comes from thermally excited electron-hole pairs of the surface and not from initial NO translational energy. Thus, it is interesting to compare the absolute excitation probabilities for $\operatorname{NO}(v=1)$ and $\mathrm{NO}(v=2)$ obtained in ref. 17 to the expected values should the NO molecules achieve complete thermalization with the surface. This thermal limit represents the maximum excitation probability possible by this mechanism as long as molecules are in thermal equilibrium with the surface. It is immediately clear that an Arrhenius dependence on $T_{\mathrm{S}}$ is incompatible with the thermal limit. However, the analytical representation of our rate equation model presented below allows us to show that the Arrhenius $T_{\mathrm{S}}$ dependence is the low $T_{\mathrm{S}}$ limit of a more general form that is compatible with the high temperature thermal limit.

We will also show that the Arrhenius pre-exponential contains interesting information that can be interpreted in terms of an overtone excitation mechanism for producing $\mathrm{NO}(v=2)$. Here we distinguish between collisions where $\mathrm{NO}(v=2)$ is produced directly from $\mathrm{NO}(v=0)$ and collisions where sequential up-pumping via $\mathrm{NO}(v=1)$ takes place. Through a proper understanding of the analytical expressions derived here, it is even possible to derive the relative importance of the overtone vs. the sequential mechanism from experimentally derived Arrhenius parameters.

\section{The rate of vibrational energy transfer}

Our approach to electronically mediated vibrational excitation and relaxation in gas-metal surface collisions is based on ideas similar or identical to those used previously to describe energy transfer in such processes as: (1) inelastic scattering of electrons from diatomic molecules, ${ }^{22,23}$ (2) lifetime broadening of vibrational lines of $\mathrm{CO}$ molecules adsorbed on a $\mathrm{Cu}(100)$ 


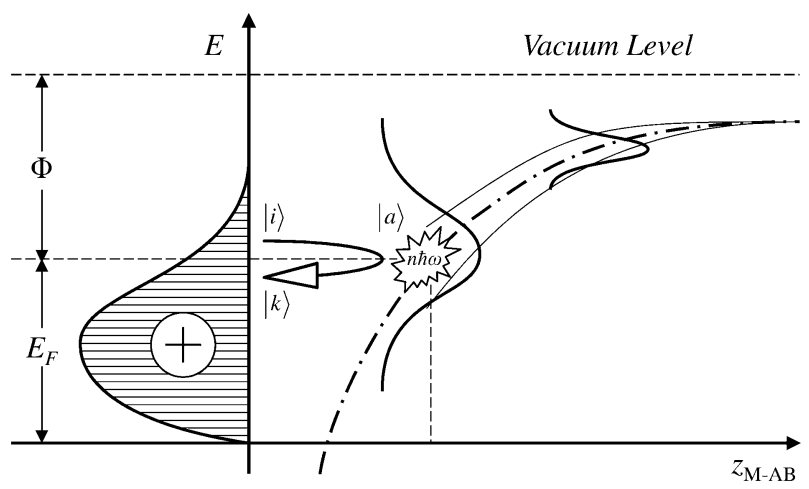

Fig. 2 Energy as a function of molecule-surface distance. Hatched area depicts conduction electrons (with the positive image charge) as the product of the Fermi-Dirac function (with the Fermi level $E_{\mathrm{F}}$ ) and the three-dimensional density of states; $\Phi$ is the work function of the metal surface; $|a\rangle$ is the stabilized and broadened affinity level; $|i\rangle$ and $|k\rangle$ are the initial and final electronic states in the metal. See Section 4.

surface, ${ }^{24,25}$ (3) atomic and molecular sticking to metal surfaces, ${ }^{26-31}$ and (4) single-quantum vibrational excitation in gas-metal collisions. ${ }^{5}$

In essence, the mechanism of energy transfer is presumed to be hopping of electrons from the metal surface to the lowest unoccupied molecular orbital (LUMO) of the molecule, which becomes energetically accessible at some critical moleculesurface distance. At this distance, the molecular affinity level $|a\rangle$ with energy $\varepsilon_{\mathrm{a}}(z)$ approaches the energy of the Fermi level due to Coulomb image charge stabilization (and broadening) as depicted in Fig. 2.

When $|a\rangle$ is energetically near the Fermi level, the molecule can form a short-lived anionic state by resonantly accommodating an electron from the surface in the LUMO. The rapid return of the electron to the metal results in lifetime broadening of $|a\rangle$, which decreases with distance from the surface. It is conceivable that such a charge transfer event can either excite or relax the vibrational degree of freedom of the molecule, depending on the precise energies of the initial $|i\rangle$ and final $|k\rangle$ states of the exchanged electron in the metal.

The overall rate $k_{v v^{\prime}}$ of such an energy transfer process was calculated by Persson and Persson ${ }^{24}$ to describe singlequantum vibrational relaxation of $\mathrm{CO}$ adsorbed on $\mathrm{Cu}(100)$ by applying the first order time dependent perturbation theory and using Fermi's Golden Rule as

$$
k_{\nu \nu^{\prime}}=\frac{2 \pi}{\hbar} \sum_{i, k}\left|\left\langle i \nu\left|H^{\prime}\right| k \nu^{\prime}\right\rangle\right|^{2} \delta\left(\varepsilon_{i}-\varepsilon_{k}-\hbar \omega_{0}\right)
$$

where $v$ and $v^{\prime}$ are initial and final vibrational states of the molecule, $\varepsilon_{i}$ and $\varepsilon_{k}$ are energies of the initial $|i\rangle$ and final $|k\rangle$ electronic states in the metal, $\omega_{0}$ is the vibrational frequency, and $H^{\prime}$ is the perturbation corresponding to the AndersonNewns type electronic Hamiltonian ${ }^{32,33}$ of the system.

Here we propose that the same basic physical mechanism governs both excitation and relaxation, and that expression (4) is valid quite generally - not only for single-quantum, but also for a direct multi-quantum vibrational energy transfer. In passing we point out that whether such a perturbation theory based approach could be used to describe the multi-quantum vibrational relaxation of ref. 34 remains an open question. Following the analysis of ref. 24 and expanding upon it to take into account the finite temperature of the surface by incorporating the Fermi-Dirac distribution function for the electrons in the conduction band of the metal, we obtain the following expression for the rate of vibrational energy transfer

$$
k_{v v^{\prime}}=\alpha_{v^{\prime} v} \frac{E_{v v^{\prime}}}{e^{E_{\nu \nu^{\prime}} / k_{\mathrm{b}} T_{\mathrm{S}}}-1} \quad \text { with } \quad \alpha_{v v^{\prime}}=\frac{2 \pi}{\hbar} \lambda_{v v^{\prime}}^{2} \rho_{\mathrm{a}}^{2}
$$

where $E_{v v^{\prime}}$ is the vibrational energy change of the molecular oscillator, $\lambda_{v v^{\prime}}$ is the coupling constant describing the interaction between the electronic occupation of the affinity level $|a\rangle$ and the vibrational degree of freedom of the molecule, and $\rho_{\mathrm{a}}$ is the density of states projected onto the molecular affinity level $|a\rangle$, $k_{\mathrm{b}}$ is the Boltzmann constant, and $T_{\mathrm{S}}$ is the temperature of the surface. See Appendix A for details.

Due to the inherent symmetry of Fermi's Golden Rule, eqn (4), with respect to the choice of the initial and final states $|i\rangle$ and $|k\rangle$, it is clear that the sign of $E_{v v^{\prime}}$ in the expression (5) determines whether it describes the rate of vibrational excitation (positive) or relaxation (negative). Likewise, the coefficients $\alpha_{v v^{\prime}}$ and $\alpha_{v^{\prime} v}$ are taken to be equal, which reflects microscopic reversibility.

Comparing eqn (1) and (5) we may relate the state-to-state kinetic model of ref. 17 to Fermi's Golden Rule. Indeed, evaluation of the sums in Fermi's Golden Rule (4) leads to integrals identical to (1) with the assumption that the coupling constant does not depend strongly on energy. In order to evaluate these analytically, the density of states functions were carried outside of the integral in eqn (5). This is justified on the basis that the density of states varies slowly with energy and can thus be treated as a constant over the energy range where electron-hole pairs involved in the energy transfer are found. Therefore, the expression for the rate constants (5) can be used within the kinetic model of vibrational energy transfer (2) introduced in ref. 17 as will be shown below.

The surface temperature dependence of the vibrational excitation (a) and relaxation (b) rates given by eqn (5) are shown in Fig. 3 as solid lines. Here, we show the case of singlequantum vibrational energy transfer between $\mathrm{NO}(v=0)$ and $\mathrm{NO}(v=1)$ with $E_{v v^{\prime}}=0.236 \mathrm{eV}$ (and $\alpha_{v v^{\prime}}=\alpha_{v^{\prime} v}=1$ for convenience).

To facilitate the analysis of expression (5), it is convenient to consider limiting cases of low and high surface temperatures, relative to the vibrational spacing, which are also shown in Fig. 3 as dash-dotted and dashed lines, respectively.

$$
E_{v v^{\prime}}>0 \quad \text { and } \quad k_{\mathrm{b}} T_{\mathrm{S}} \ll E_{v v^{\prime}} \rightarrow k_{v v^{\prime}} \approx \alpha_{v v^{\prime}} E_{v v^{\prime}} e^{-\frac{E_{v^{\prime} v}}{k_{\mathrm{b}} T_{\mathrm{S}}}}
$$

$$
\begin{aligned}
& E_{v v^{\prime}}>0 \text { and } \\
& k_{\mathrm{b}} T_{\mathrm{S}} \gg E_{v v^{\prime}} \rightarrow k_{v v^{\prime}} \approx \alpha_{v v^{\prime}} k_{\mathrm{b}} T_{\mathrm{S}}-\frac{\alpha_{v v^{\prime}} E_{v v^{\prime}}}{2}+\frac{\alpha_{v v^{\prime}} E_{v v^{\prime}}^{2}}{12 k_{\mathrm{b}} T_{\mathrm{S}}}
\end{aligned}
$$




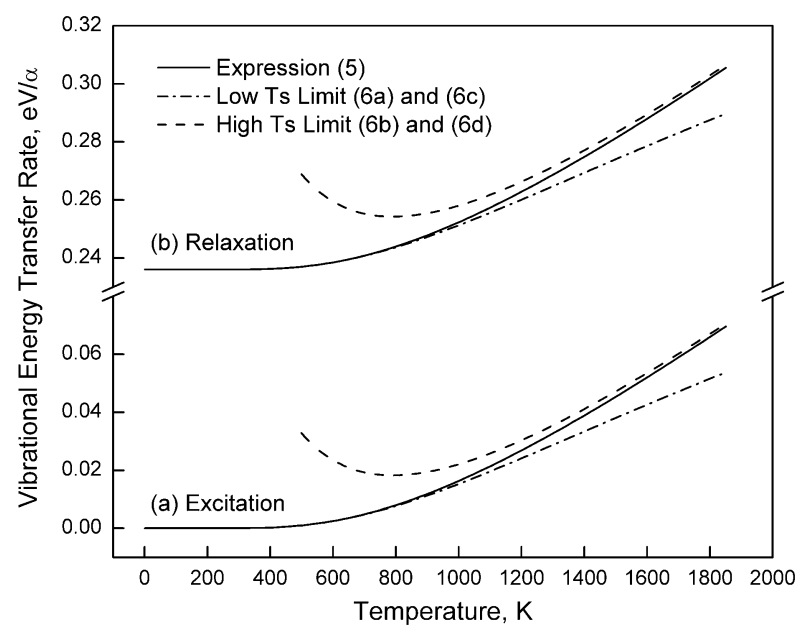

Fig. 3 Temperature dependence of the vibrational energy transfer rates given by the expression (5) (solid lines) and its approximations (6a)-(6d) for low (dash-dot line) and high (dashed line) temperature limits. Note the clear departure from the Arrhenius form at $\sim 1100 \mathrm{~K}$.

$$
\begin{gathered}
E_{v v^{\prime}}<0 \text { and } \\
k_{\mathrm{b}} T_{\mathrm{S}} \ll E_{v v^{\prime}} \rightarrow k_{v^{\prime} v} \approx \alpha_{v v^{\prime}} E_{v v^{\prime}}\left(1+e^{-\frac{E_{v^{\prime} v}}{k_{\mathrm{b}} T_{\mathrm{S}}}}\right) \\
E_{v v^{\prime}}<0 \text { and } \\
k_{\mathrm{b}} T_{\mathrm{S}} \gg E_{v v^{\prime}} \rightarrow k_{v^{\prime} v} \approx \alpha_{v v^{\prime}} k_{\mathrm{b}} T_{\mathrm{S}}+\frac{\alpha_{v v^{\prime}} E_{v v^{\prime}}}{2}+\frac{\alpha_{v v^{\prime}} E_{v v^{\prime}}^{2}}{12 k_{\mathrm{b}} T_{\mathrm{S}}}
\end{gathered}
$$

Eqn (6) are obtained by approximating the denominator in (5) appropriately (e.g. by comparing the exponential term to unity and using its Taylor series expansion) and setting $E_{v v^{\prime}}=\left|E_{v v^{\prime}}\right|$ so that it is always positive (here and through the rest of the equations in the paper).

As is clear from Fig. 3, the limiting low temperature case is well described by an Arrhenius form given by (6a) and (6c) for the rates of excitation and relaxation, respectively. In the event of vibrational excitation with $E_{v v^{\prime}}>0$, the rate given by (5) and approximated by (6a) vanishes at zero surface temperature - in accord with the idea of thermally excited electron-hole pairs being the source and mediator of the vibrational energy transfer-and grows in the Arrhenius fashion with surface temperature. In contrast, the rate of vibrational relaxation given by (5) with $E_{v v^{\prime}}<0$ and approximated by (6c) converges to a non-zero value when $T_{\mathrm{S}}$ approaches zero, which is identical to the result obtained by Persson and Persson. ${ }^{24}$

At higher temperatures $\left(T_{\mathrm{S}}>1100 \mathrm{~K}\right)$ the approximate Arrhenius form begins to fail, as it leads to saturation, while the complete expression (5) exhibits the expected continuing linear growth (with the slope independent of the vibrational energy $E_{v v^{\prime}}$ ) of both excitation and relaxation rates, see Fig. 3, and is well approximated by (6b) and (6d) for excitation and relaxation, respectively. Note that the inverse temperature term in (6b) and (6d) vanishes in the high temperature limit. For the discussion of the highest temperature where expression (5) is still valid, see Appendix A.

Expression (5) suggests the same functional form of the temperature dependence for both excitation and relaxation rates with a temperature independent difference between the two. Indeed, it can be immediately obtained from (5) that

$$
k_{v^{\prime} v}-k_{v v^{\prime}}=\alpha E_{v v^{\prime}}
$$

Essentially, this result originates from the symmetry of the Fermi-Dirac distributions for the electrons (promoting excitation) and corresponding holes (facilitating relaxation) as the number of both grows with the temperature in the same manner. This result suggests another interesting experiment, namely to test if this intrinsic and simple relationship between the quantitative excitation and relaxation probabilities can be observed.

Another appealing feature of the expression for the vibrational energy transfer rates (5) is that the excitation and relaxation rate constants it prescribes satisfy the principle of detailed balance. As is readily seen from (5), the ratio of the rates yields the Boltzmann factor:

$$
\frac{k_{v v^{\prime}}}{k_{v^{\prime} v}}=e^{-\frac{E_{v v^{\prime}}}{k_{\mathrm{b}} T_{\mathrm{S}}}}
$$

This property of the analytical expression for the vibrational energy transfer rates (5) establishes a condition necessary for a system of rate equations describing the kinetics of processes (2) to provide solutions that approach thermal equilibrium at infinite interaction time. Thus, the essential identity between (1) and (5) that was already mentioned above is the reason why the numerical solutions of (2) demonstrated by Cooper et al. ${ }^{17}$ lead to thermal equilibrium at infinite time.

\section{Kinetics and absolute excitation probabilities}

One of the major ideas leading to the kinetic model introduced in our previous paper on the subject ${ }^{17}$ is that the absolute vibrational excitation probability is determined by a competition of simultaneous excitation and relaxation events occurring during the interaction time while the molecules stay in the interaction region in the vicinity of the surface. This approach led to construction of a system of linear differential rate equations in the form of (2), the solution of which was used to describe the time and surface temperature dependence of the experimentally observed excitation probabilities. Here we revisit this kinetic approach, this time using the analytical expression for the rate constants (5) introduced in this work. This allows us to evaluate features of direct overtone and sequential mechanisms in $\mathrm{NO}(v=0 \rightarrow 1,2)$ multi-quantum vibrational excitation and compare to experiment.

\subsection{Single-step excitation: $\mathrm{NO}(v=0 \rightarrow 1)$ and $\mathrm{NO}(v=0 \rightarrow 2)$}

In order to kinetically describe a single-step mechanism of vibrational excitation, we first consider a dynamic process where ground state $\mathrm{NO}(v=0)$ molecules are continuously excited with the rate $k_{01}$ to the first vibrational state $\operatorname{NO}(v=1)$, and where excited state molecules relax with the rate $k_{10}$ back down to the ground state:

$$
\mathrm{NO}(v=0) \stackrel{k_{01}, k_{10}}{\longleftrightarrow} \mathrm{NO}(v=1)
$$


The differential rate equations for such a process can be written in the form (2) as

$$
\mathrm{d} n_{0} / \mathrm{d} t=k_{10} n_{1}-k_{01} n_{0}, \mathrm{~d} n_{1} / \mathrm{d} t=k_{01} n_{0}-k_{10} n_{1}
$$

where $n_{0}$ and $n_{1}$ are the numbers of molecules in the ground and excited states, respectively. Solutions of (10) can be immediately obtained analytically, leading to the following expression for the $n_{1}(t) / n_{0}(t=0)$ ratio observed in the experiments:

$$
\frac{n_{1}(t)}{n_{0}(t=0)}=\frac{k_{01}}{k_{01}+k_{10}}\left(1-e^{-\left(k_{01}+k_{10}\right) t}\right)
$$

Now we can substitute expression (5) derived above for the rates $k_{01}$ and $k_{10}$. This leads to eqn (12), which gives the absolute vibrational excitation probability $P_{v v^{\prime}}$ as a function of the interaction time $t$ and surface temperature $T_{\mathrm{S}}$ :

$$
P_{v v^{\prime}}=\frac{n_{1}(t)}{n_{0}(t=0)}=\frac{1-e^{-\alpha_{v v^{\prime}} E_{v v^{\prime}} \operatorname{coth}\left(\frac{E_{v v^{\prime}}}{2 k_{\mathrm{b}} T_{\mathrm{S}}}\right) t}}{1+e^{\frac{E_{v v^{\prime}} T_{\mathrm{S}}}{k^{\prime}}}}
$$

To discuss the properties of expression (12) it is again convenient to examine the limiting cases of short and long interaction times and low and high surface temperatures. Using the same approximations for the exponential terms as in (6a)-(6d) and by recognizing that the coth function can be represented by the inverse of its argument (the first term of its Taylor series expansion) and unity in cases of high and low temperatures, respectively, we obtain

$$
\begin{aligned}
& t \ll 1 / k_{v v^{\prime}} \text { and } \\
& k_{\mathrm{b}} T_{\mathrm{S}} \ll E_{v v^{\prime}} \rightarrow P_{v v^{\prime}} \approx k_{v v^{\prime}} t=\alpha_{v v^{\prime}} E_{v v^{\prime}} t e^{-\frac{E_{v v^{\prime}}}{k_{\mathrm{b}} T_{\mathrm{S}}}} \\
& t \ll 1 / k_{v v^{\prime}} \text { and } \\
& k_{\mathrm{b}} T_{\mathrm{S}} \gg E_{v v^{\prime}} \rightarrow P_{v v^{\prime}} \approx k_{v v^{\prime}} t \\
& =\left(\alpha_{v v^{\prime}} k_{\mathrm{b}} T_{\mathrm{S}}-\frac{\alpha_{v v^{\prime}} E_{v v^{\prime}}}{2}+\frac{\alpha_{v v^{\prime}} E_{v v^{\prime}}^{2}}{12 k_{\mathrm{b}} T_{\mathrm{S}}}\right) t \\
& t \rightarrow \infty \quad \text { and } \quad k_{\mathrm{b}} T_{\mathrm{S}} \ll E_{v v^{\prime}} \rightarrow P_{v v^{\prime}} \approx e^{-\frac{E_{v v^{\prime}} T_{\mathrm{S}}}{k_{\mathrm{S}} T^{\prime}}} \\
& t \rightarrow \infty \quad \text { and } \quad k_{\mathrm{b}} T_{\mathrm{S}} \gg E_{v v^{\prime}} \rightarrow P_{v v^{\prime}} \approx 1 / 2
\end{aligned}
$$

The physical meaning of these results can be immediately understood. In the limits of short interaction times and low surface temperature, the excitation probability given by (12) is well approximated by an Arrhenius form of (13a) - see Fig. 4 where the exact solution (12) is plotted together with approximations (13a) and (13b) as a function of temperature for three arbitrarily chosen short interaction times. As the temperature of the surface becomes larger, the Arrhenius behavior breaks down, and the linear temperature dependence takes over, according to approximation (13b).

The pre-exponent of this Arrhenius form, as essentially given by the numerator of (12), is almost independent of the temperature of the surface, and its dependence on the interaction time is shown in Fig. 5. The pre-exponent starts off linearly at short times as suggested by (13a) and approaches a limiting value of unity given by (13c) as the interaction time

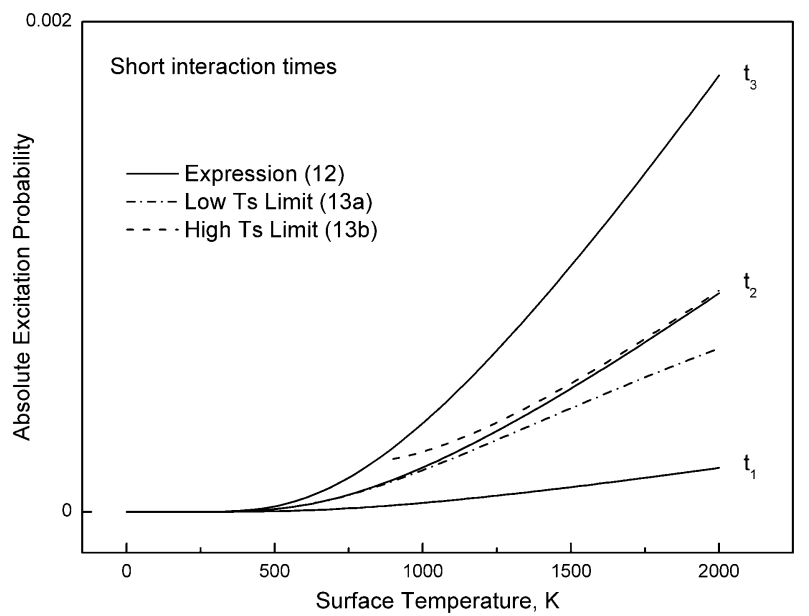

Fig. 4 Vibrational excitation probability of the single-step mechanism (9) as a function of surface temperature (low $T_{\mathrm{S}}$ and short $t$ limit) for three arbitrary $t_{1}<t_{2}<t_{3}$. Solid line: the exact solution (12); dash line: high temperature limit (13b); dash-dot line: low temperature limit. Note that the Arrhenius form breaks down at temperatures $T_{\mathrm{S}}>1100 \mathrm{~K}$.

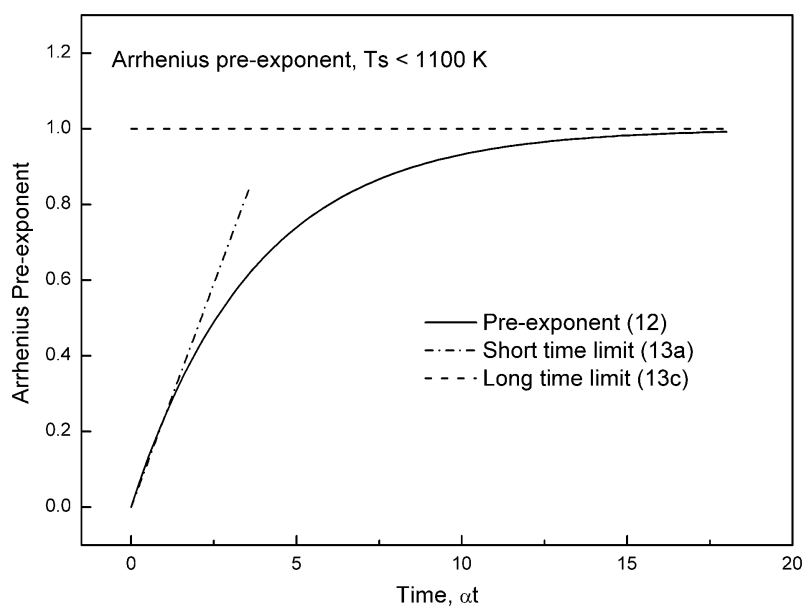

Fig. 5 Dependence of the low temperature limit Arrhenius preexponent of the vibrational excitation probability of the single-step mechanism (9) on the interaction time. At short interaction times the linear growth from zero is given by (13a), followed by saturation to unity given by (12).

goes to infinity. This limiting value represents the system coming to thermal equilibrium.

Fig. 6 and 7 illustrate the approach to thermal equilibrium where the exact solutions, eqn (12), are plotted as a function of the interaction time and surface temperature, respectively, approaching the maximum possible value of $\frac{1}{2}$ given by (13d).

It is thus clear that the Arrhenius surface temperature dependence of the single-quantum vibrational excitation probability observed experimentally ${ }^{1,17-19}$ can be reproduced by a kinetic model (9)-(10) with the analytical expression (5) for the rate of electronically mediated vibrational energy transfer introduced in this work.

Considering the specific case of NO molecules with $E_{01}=0.236 \mathrm{eV}$ and the experimentally studied surface temperature range from 300 to $1000 \mathrm{~K}$ the condition $k_{\mathrm{b}} T_{\mathrm{S}} \ll E_{01}$ is fulfilled, expression (12) is well approximated by (13a) giving 


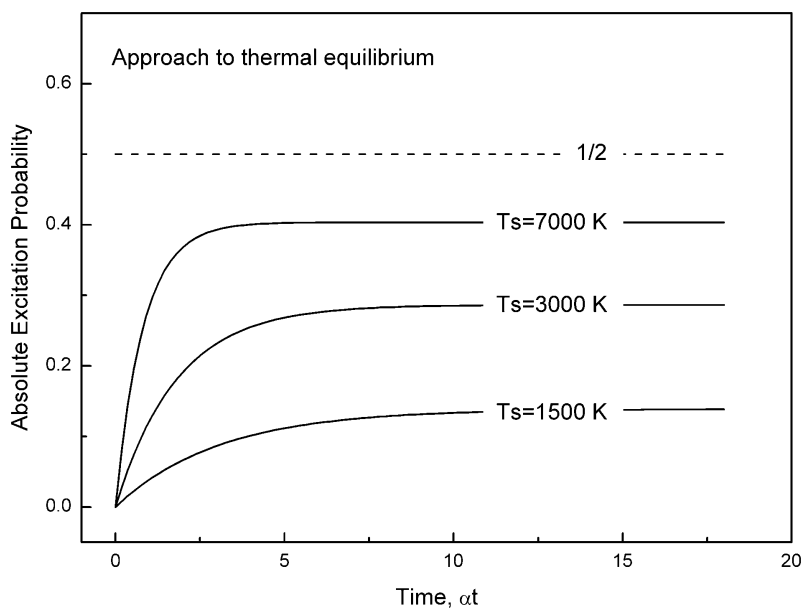

Fig. 6 Vibrational excitation probability of the single-step mechanism, (9) as a function of interaction time for several surface temperaturesapproach to thermal equilibrium. Solid lines: exact solutions (12) plotted for several arbitrary temperatures; dash line: maximum thermal equilibrium value at infinite surface temperature (13d).

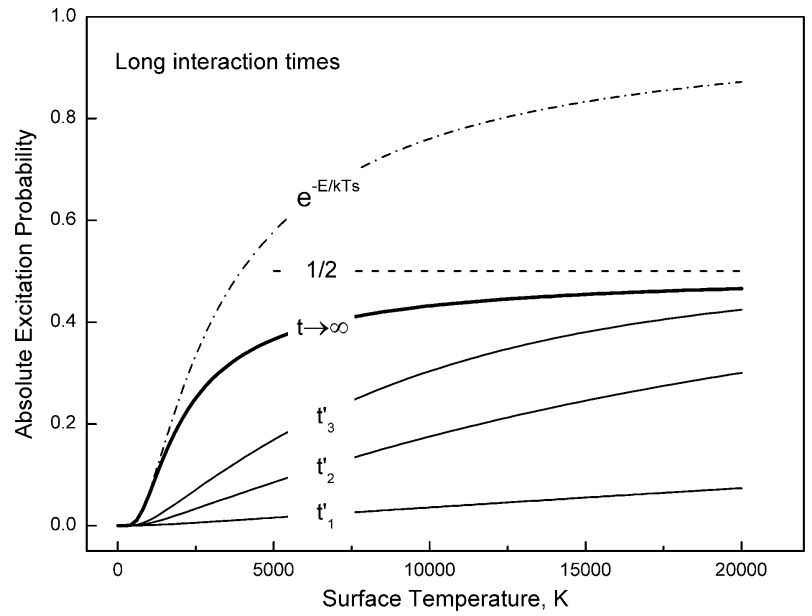

Fig. 7 Vibrational excitation probability of a single-step mechanism (9) as a function of surface temperature for several interaction timesapproach to thermal equilibrium. Solid lines: exact solutions (12); dash-dot line: Arrhenius form at infinite time (13c); dash line: maximum thermal equilibrium value (13d).

rise to Arrhenius behavior in the surface temperature dependence, see Fig. 1. To see deviations from the Arrhenius behavior, temperatures higher than $1100 \mathrm{~K}$ would be required.

As the expression for the vibrational energy transfer rate (5) is postulated to be valid for both single- and multi-quantum excitation, we conclude that expression (12) for the absolute excitation probability can be directly applied to the case of single-step direct overtone $\mathrm{NO}(v=0 \rightarrow 2)$ channel with the vibrational energy $E_{v v^{\prime}}$ taken as $E_{02}=2 \times \hbar \omega_{0}$ and the corresponding coefficient $\alpha_{v v^{\prime}}=\alpha_{02}$. Clearly, all expressions for the limiting cases (13a) and (13b) hold for the overtone channel as well, and one can expect (as seen in Fig. 1 experimentally) to obtain an Arrhenius dependence on surface temperature with the slope (effective activation energy) equal to two vibrational quanta, and with the pre-exponent ranging from zero to unity depending on the interaction time.

\subsection{Sequential two-step excitation: $\mathrm{NO}(v=0 \rightarrow 1 \rightarrow 2)$}

Multi-quantum vibrational excitation $\mathrm{NO}(v=0 \rightarrow 2)$ observed in the experiments can occur not only directly via the overtone excitation mechanism mentioned above, but also sequentially by two single-quantum transitions through an intermediate $\mathrm{NO}(v=1)$ state. In order to compare both mechanisms, we now briefly consider the kinetics of a two-step process, such as

$$
\mathrm{NO}(v=0) \stackrel{k_{01}, k_{10}}{\longleftrightarrow} \mathrm{NO}(v=1) \stackrel{k_{12}, k_{21}}{\longleftrightarrow} \mathrm{NO}(v=2)
$$

Here we shall assume that the rates of single-quantum vibrational excitation and relaxation processes $\mathrm{NO}(v=0 \rightarrow 1)$ and $\mathrm{NO}(v=1 \rightarrow 2)$ are equal, that is $k_{12}=k_{01}$ and $k_{21}=k_{10}$, which is justified by the fact that derivation of the expressions for the rates (4) and (5) does not depend on the specific choice of initial and final vibrational states. In this case the system of differential rate equations written in the form (2) is given by

$$
\begin{aligned}
& \mathrm{d} n_{0} / \mathrm{d} t=k_{10} n_{1}-k_{01} n_{0} \\
& \mathrm{~d} n_{1} / \mathrm{d} t=k_{01} n_{0}-k_{10} n_{1}-k_{01} n_{1}+k_{10} n_{2} \\
& \mathrm{~d} n_{2} / \mathrm{d} t=k_{01} n_{1}-k_{10} n_{1}
\end{aligned}
$$

As in the previous case of direct single-step vibrational excitation, the solutions of (15) provide analytical expressions for the absolute vibrational excitation probabilities, but unlike (12) these are too ungainly to be shown here explicitly. Instead, we will consider just the limiting case of low temperatures (as it corresponds to our experimental conditions) for short and long interaction times:

$$
\begin{aligned}
& t \ll 1 / k_{01} \quad \text { and } \\
& k_{\mathrm{b}} T_{\mathrm{S}} \ll E_{01} \rightarrow P_{02} \approx \frac{\left(k_{01} t\right)^{2}}{2}=\frac{1}{2}\left(\alpha_{01} E_{01} t\right)^{2} e^{-\frac{2 E_{01}}{k_{\mathrm{b}} T_{\mathrm{S}}}} \\
& t \rightarrow \infty \text { and } k_{\mathrm{b}} T_{\mathrm{S}} \ll E_{01} \rightarrow P_{02} \approx e^{-\frac{2 E_{01}}{k_{\mathrm{b}} T_{\mathrm{S}}}}
\end{aligned}
$$

As we can see, in the case of the sequential $\mathrm{NO}(v=0 \rightarrow 1 \rightarrow 2)$ excitation mechanism, an Arrhenius form with the activation energy $E_{\mathrm{a}}=2 \times E_{01}=2 \times \hbar \omega_{0}$ and time dependent pre-exponent ranging from zero to unity for the vibrational excitation probability as a function of surface temperature is expected. Therefore, the experimentally observed Arrhenius slope alone cannot be used to distinguish between the direct overtone and the sequential, two-step mechanisms. On the other hand, the pre-exponent of the sequential processes is expected to be quite different for the two mechanismscompare (13a) and (16a) - and can be used to reveal the excitation mechanism, provided we have some information on the relationship between coefficients $\alpha_{01}$ and $\alpha_{02}$, a condition that we will show below is fulfilled.

\subsection{Full model and fit to experimental data}

By allowing both direct single- and two-step sequential excitation and relaxation processes to proceed concurrently, we can assemble a system of differential rate equations like shown 
above in (2) that combines all possible excitation and relaxation pathways:

$$
\begin{aligned}
& \mathrm{d} n_{0} / \mathrm{d} t=k_{10} n_{1}-k_{01} n_{0}-k_{02} n_{0}+k_{20} n_{2} \\
& \mathrm{~d} n_{1} / \mathrm{d} t=k_{01} n_{0}-k_{10} n_{1}-k_{12} n_{1}+k_{21} n_{2} \\
& \mathrm{~d} n_{2} / \mathrm{d} t=k_{12} n_{1}-k_{21} n_{2}+k_{02} n_{0}-k_{20} n_{2}
\end{aligned}
$$

All rate constants $k_{v v^{\prime}}$ in (17) are given by the analytical expression (5), and with the already mentioned assumptions of $\alpha_{v v^{\prime}}=\alpha_{v^{\prime} v}$ (due to microscopic reversibility) and $\alpha_{01}=\alpha_{12}$ (due to independence of the rates on the initial vibrational state) can be written as

$$
\begin{aligned}
& k_{01}=k_{12}=\frac{\hbar \omega_{0}}{e^{\hbar \omega_{0} / k_{\mathrm{b}} T_{\mathrm{S}}}-1}, k_{10}=k_{21}=\frac{\hbar \omega_{0}}{1-e^{-\hbar \omega_{0} / k_{\mathrm{b}} T_{\mathrm{S}}}}, \\
& k_{02}=\beta \frac{2 \hbar \omega_{0}}{e^{2 \hbar \omega_{0} / k_{\mathrm{b}} T_{\mathrm{S}}}-1}, k_{20}=\beta \frac{2 \hbar \omega_{0}}{1-e^{-2 \hbar \omega_{0} / k_{\mathrm{b}} T_{\mathrm{S}}}}
\end{aligned}
$$

where coefficients $\alpha_{v v^{\prime}}$ are taken as follows:

$$
\alpha_{01}=\alpha_{10}=\alpha_{12}=\alpha_{21}=1, \alpha_{02}=\alpha_{20}=\beta \times \alpha_{01}
$$

and the factor $\beta$ accounts for the relative importance of direct single-step $\mathrm{NO}(v=0 \rightarrow 1)$ and overtone $\mathrm{NO}(v=0 \rightarrow 2)$ excitation. With such a choice of numerical values for the coefficients $\alpha_{v v^{\prime}}$ the solutions of (17) will be expressed in units of time $\tau$ given by

$$
\tau=\alpha_{01} t
$$

Thus, with this approach the system of rate equations (17) has just two unknown parameters, interaction time $\tau$ and coupling strength ratio $\beta$, and both of these can be obtained by comparing the solutions of (17) to the experimentally obtained surface temperature dependence of the absolute vibrational excitation probabilities of $\mathrm{NO}(v=1)$ and $\mathrm{NO}(v=2)$.

In order to carry out such a fitting procedure, we first solve the system of rate equations (17) to find the solutions as functions $n_{0}\left(\tau, T_{\mathrm{S}}, \beta\right), n_{1}\left(\tau, T_{\mathrm{S}}, \beta\right)$ and $n_{2}\left(\tau, T_{\mathrm{S}}, \beta\right)$ that can be used to obtain corresponding excitation probabilities $P_{1}\left(\tau, T_{\mathrm{S}}, \beta\right)$ and $P_{2}\left(\tau, T_{\mathrm{S}}, \beta\right)$ as

$$
P_{1}=\frac{n_{1}\left(\tau, T_{\mathrm{S}}, \beta\right)}{n_{0}\left(\tau=0, T_{\mathrm{S}}, \beta\right)} \quad \text { and } \quad P_{2}=\frac{n_{2}\left(\tau, T_{\mathrm{S}}, \beta\right)}{n_{0}\left(\tau=0, T_{\mathrm{S}}, \beta\right)}
$$

and then perform a global least squares fit on two experimental data sets simultaneously using the same pair of $\tau$ and $\beta$ as the adjustable parameters. This yields

$$
\tau_{\mathrm{eff}}=1.94(1 / \mathrm{eV}) \text { and } \beta=0.68
$$

The best fit results, $P_{1}\left(\tau, T_{\mathrm{S}}, \beta\right)$ and $P_{2}\left(\tau, T_{\mathrm{S}}, \beta\right)$ using the values (22), are shown with the experimental data in Fig. 1 as solid lines. As expected (see discussion of (13a) and (16a) above), the temperature dependence of excitation probabilities of both vibrational states obtained from the global fit is practically indistinguishable from the canonical Arrhenius fit (shown in Fig. 1 as dash-dot line), especially given the scatter in the presently available experimental data points.
As noted above, the numerical values of the effective interaction time $\tau$ and the ratio $\beta$ of coefficients (22) obtained in this work are in a perfect agreement with those reported earlier. ${ }^{17}$

Here we would like to point out that although the model has two adjustable parameters - interaction time $\tau_{\text {eff }}$ and coefficient ratio $\beta$ - the fitting procedure does not allow for much variability in the choice of these constants. Indeed, the numbers for $n_{1}\left(\tau, T_{\mathrm{S}}, \beta\right)$ that represents $\mathrm{NO}(v=1)$ excitation probability are very insensitive to the value of the parameter $\beta$-because $n_{1}\left(\tau, T_{\mathrm{S}}, \beta\right)$ is dominated by the direct production of $\operatorname{NO}(v=1)$ from $\mathrm{NO}(v=0)$. Therefore, the interaction time $\tau_{\text {eff }}$ can be obtained with rather good precision assuming $\beta=1$ from the $P_{1}\left(T_{\mathrm{S}}\right)$ dataset alone, and then the value of the factor $\beta$ can be adjusted using the $P_{2}\left(T_{\mathrm{S}}\right)$ dataset. This procedure can be repeated recursively. We found that $\tau_{\text {eff }}$ and $\beta$ obtained with just a single iteration are already within a few percent of the values (22), enabling a very robust and easy global fitting procedure.

\section{Discussion}

As shown above, the analytical expression (5) for the rates of electronically mediated vibrational energy transfer introduced in this work can be used to describe single- and multi-quantum vibrational excitation of $\mathrm{NO}$ at $\mathrm{Au}(111)$ within the framework of the kinetic model described earlier ${ }^{17}$ and revisited in this work. Indeed, expression (5) conforms to the expected values (zero and a constant, respectively) for these rates at zero surface temperature, it correctly describes the ratio of the excitation and relaxation rates to satisfy the principle of detailed balance (8), and when used with the kinetic model it provides a very good fit to all of the available experimental data.

One of the most important features of expression (5) is that (together with the kinetic model) it allows physical interpretation of the Arrhenius form of the surface temperature dependence, in particular, the pre-exponential factor, that has long been an empirical hallmark of electronically non-adiabatic vibrational energy transfer. Specifically, we may now state that Arrhenius behavior is an approximation of a more general solution, eqn (12), in the limit of low surface temperatures, relative to vibrational spacing, see (13a) and (13c). Although the exponential term with the activation energy equal to the difference in energy between the vibrational states has previously been suggested to arise from the thermal distribution of electron-hole pairs in the metal, ${ }^{1}$ the nature of the pre-exponential factor remained largely unexplored. Kinetic analysis of the elementary excitation processes (9) and (14) empowered by expression (5) carried out in this work not only shows explicitly that the Arrhenius pre-exponential factor may only assume values between zero and unity, but also provides its functional dependence on vibrational energy $E_{v v^{\prime}}$, interaction time $t$, and electronic-vibrational coupling $\lambda_{v v^{\prime}}$ with the density of states $\rho_{\mathrm{a}}$ via the coefficient $\alpha_{v v^{\prime}}$ for both direct and sequential excitation mechanisms in the limit of short interaction times, see (13a) and (16a).

This newly obtained knowledge on the Arrhenius preexponential factors can be used at once to infer deeper understanding of the mechanism of the observed $\mathrm{NO}(v=0 \rightarrow 2)$ 
vibrational excitation. Indeed, it immediately follows from comparison of (13a) and (16a) that if $\mathrm{NO}(v=2)$ is predominantly populated by a sequential mechanism, its Arrhenius pre-exponent $A_{012}$ is expected to be of the order of

$$
A_{012}=\frac{1}{2}\left(A_{01}\right)^{2}=\frac{1}{2}(0.38)^{2}=0.07
$$

This value compares very poorly with the value of 0.46 derived from experiment. See Fig. 1 and its discussion above. Thus, this result indicates that most of the excited $\mathrm{NO}(v=2)$ molecules are produced via the direct overtone excitation mechanism. This remarkably simple analysis provides an elegant way to interpret the $T_{\mathrm{S}}$ dependence of vibrational excitation in a highly detailed mechanistic fashion.

If direct overtone excitation dominates, both pre-exponents $A_{01}$ and $A_{02}$ will be given by (13a), and from their ratio we can estimate the relative magnitude of the coefficients $\alpha_{01}$ and $\alpha_{02}$ as

$$
\frac{\alpha_{02}}{\alpha_{01}}=\frac{1}{2} \frac{A_{02}}{A_{01}}=\frac{1}{2} \times \frac{0.46}{0.38} \approx 0.6
$$

This number compares very well with the value of 0.68 obtained for the same ratio from the global fit of the solutions of the full kinetic model to the experimental temperature dependence (22).

The small difference between the two values is likely due to the fact that both excitation mechanisms (sequential and direct overtone) contribute to the production of $\mathrm{NO}(v=2)$. The same line of analysis can be pushed a step further to roughly estimate relative contributions of the direct overtone and sequential single-quantum mechanisms to the entire amount of the excited $\mathrm{NO}(v=2)$ molecules measured in the experiments. Let $x$ and $y$ be the corresponding fractions such that $x+y=1$, and assuming interaction times are not too long we can use expressions (13a) and (16a) to obtain the total amount of $\mathrm{NO}(v=2)$ as a sum

$$
P_{02}=x k_{02} t+y \frac{\left(k_{01} t\right)^{2}}{2}=\left[x \alpha_{02} t 2 \hbar \omega_{0}+\frac{y}{2}\left(\alpha_{01} t \hbar \omega_{0}\right)^{2}\right] e^{-\frac{2 \hbar \omega_{0}}{k_{\mathrm{b}} T_{\mathrm{S}}}}
$$

Thus, the experimentally derived pre-exponent $A_{02}$ and $A_{01}$ are, respectively, given (from (13a) alone) by

$$
x \alpha_{02} t 2 \hbar \omega_{0}+\frac{y}{2}\left(\alpha_{01} t \hbar \omega_{0}\right)^{2}=0.46 \text { and } \alpha_{01} t \hbar \omega_{0}=0.38
$$

and their ratio

$$
\frac{0.46}{0.38}=2 \frac{\alpha_{02}}{\alpha_{01}} x+\frac{0.38}{2} y
$$

Using the value for $\alpha_{02} / \alpha_{01}=0.68$ from (22) and solving (27) together with $x+y=1$, we easily obtain the relative fractions of the direct and sequential contributions to be, respectively

$$
x \approx 0.86 \text { and } y \approx 0.14
$$

This result quantifies the suggested ${ }^{17}$ dominance of the singlestep overtone process over the sequential single-quantum mechanism of $\mathrm{NO}(v=2)$ vibrational excitation.
The kinetic approach explored in this work has the potential to become a general procedure for the analysis of experimental data on electronically non-adiabatic vibrational energy transfer for many systems. Especially attractive is the possibility to use the effective interaction time $\tau_{\text {eff }}$ obtained from fitting the solutions of the appropriate rate equations such as (10), (15), or (17) to the experimental data together with an independent estimate for the interaction time to calculate the numerical values of the coefficients $\alpha_{v v^{\prime}}$-which, in turn, provide a link to estimating such fundamental properties of the studied molecule-surface system as coupling between the electronic occupation of LUMO and vibrational degree of freedom $\lambda_{v v^{\prime}}$ and the density of electronic states in the metal projected onto the molecular affinity level $\rho_{\mathrm{a}}$. For example, using the value of turnaround time over twice the critical distance $t_{\mathrm{eff}}=400 \mathrm{fs},{ }^{35}$ using (20) and (22) we obtain

$$
\alpha_{01}=4.85 \times 10^{12} \text { and } \alpha_{02}=3.16 \times 10^{12}(\mathrm{eV} \times \mathrm{s})^{-1}
$$

and then, from the definition of $\alpha_{v v^{\prime}}$ given by (5)

$$
\lambda_{01} \rho_{\mathrm{a}}=0.065 \text { and } \lambda_{02} \rho_{\mathrm{a}}=0.044
$$

Although additional work is required to draw conclusions about the magnitude of these numbers, we remark that it is useful that they are accessible within the kinetic analysis employing the newly obtained expression (5) for the vibrational transfer rates.

It is clear that the suggested general validity of the ideas introduced in this work has to be tested further on as many molecule-surface systems as possible. The electronically non-adiabatic vibrational excitation data available for $\mathrm{NO}$ on two other metals- $\mathrm{Ag}(111)^{1}$ and $\mathrm{Cu}(110)^{19}$ - are already controversial, as the reported excitation probabilities exhibit Arrhenius pre-exponents larger than unity: 2.0 for $\mathrm{NO} / \mathrm{Ag}$ and 1.3 for $\mathrm{NO} / \mathrm{Cu}$. According to the results of the kinetic analysis above this would not be possible, as it implies the observed populations of vibrationally excited product were larger than the limit of thermal equilibrium with the surface. It is worth noting that absolute excitation probability measurements are remarkably difficult, and that this discrepancy may be due to experimental problems. Furthermore, for the NO case the experimental error bars would bring the data into the agreement with the thermal limit. Alternatively it might be that a certain fraction of the vibrational excitation results from a translational to vibrational mechanism. More experiments are surely needed to resolve this disagreement and to possibly refine the proposed model.

In conclusion, we remark that the theoretical framework introduced in this work allows for straightforward extension to the case where electronically non-adiabatic coupling parameters going into the rate constants expression (5) could be considered to be a function of molecule-surface distance or other degrees of freedom. This would make it possible to recalculate the solutions of the appropriate kinetic rate equations as an integral over molecular trajectories on a given potential energy surface, which would further improve the realism of the numerical values obtained from the analysis of the experimental data. 


\section{Summary}

The kinetic model describing single- and multi-quantum vibrational excitation of molecular beams of NO scattering from $\mathrm{Au}(111)$ metal surfaces introduced in ref. 17 has been further developed. An analytical expression for the rate of electronically non-adiabatic vibrational energy transfer was derived within the first order perturbation theory that is using Fermi's Golden Rule with a Newns-Anderson model Hamiltonian for the molecule-surface system. This expression was studied as a function of surface temperature in the limiting cases of low and high temperatures yielding physically meaningful results. Specifically, it predicts the same functional form of the temperature dependence for both excitation and relaxation rates with a temperature independent difference between them. It was also shown to exhibit rates that satisfy the principle of detailed balance. Furthermore, the newly introduced expression allowed for an entirely analytical analysis of the basic single- and two-step sequential kinetic processes, from which the Arrhenius form of surface temperature dependence of the absolute vibrational excitation probability was shown to emerge at the limit of low (compared to the transferred vibrational energy) temperatures. The pre-exponents of such Arrhenius functions were shown to assume numerical values within the range from zero to unity being a function of the effective interaction time, also derived in this work - which allowed us to estimate the relative contributions of direct overtone and sequential single-step mechanism of vibrational excitation of $\mathrm{NO}(v=2)$ to be $84 \%$ and $16 \%$, respectively - consistent with the conclusions of the previous analysis. ${ }^{17}$ The analytical expression for the vibrational transfer rates was employed in the numerical evaluation of the full kinetic model allowing for a combination of both direct and sequential single- and multi-quantum vibrational excitation providing an excellent fit to the experimental data and yielding a pathway to obtain numerical values for the strength of molecule-surface electronically non-adiabatic coupling.

\section{Appendix A}

In essence, the evaluation of Fermi's Golden rule for electronic transitions coupled to the molecular vibrational degree of freedom described here resembles very closely the line of reasoning employed by Persson and Persson to calculate the rate of vibrational relaxation of $\mathrm{CO}$ adsorbed on $\mathrm{Cu}(100){ }^{24}$ According to this, the rate of transitions between the initial $|i\rangle$ and final $|k\rangle$ electronic states is

$$
\Gamma=\frac{2 \pi}{\hbar}\left|\left\langle i v\left|H^{\prime}\right| k v^{\prime}\right\rangle\right|^{2} \delta\left(\varepsilon_{i}-\varepsilon_{k}-E_{v v^{\prime}}\right)
$$

where $v$ and $v^{\prime}$ are the initial and final vibrational states of the molecule, $\varepsilon_{i}$ and $\varepsilon_{k}$ are energies of the initial $|i\rangle$ and final $|k\rangle$ electronic states, $E_{v v^{\prime}}=\hbar \omega_{0} \times\left(v^{\prime}-v\right)$ is the vibrational energy change, and $H^{\prime}$ is the perturbation corresponding to the Newns-Anderson electronic Hamiltonian ${ }^{32,33}$ of the system. Designating the matrix element of the coupling between the electronic occupation of the molecular LUMO and its vibrational degree of freedom as $\lambda_{v v^{\prime}}=|<i v| H^{\prime}\left|k v^{\prime}>\right|$, (A1) can be re-written as

$$
\Gamma=\frac{2 \pi}{\hbar} \lambda_{v v^{\prime}}^{2} \delta\left(E_{i}-E_{k}-E_{v v^{\prime}}\right)
$$

The total rate of vibrational energy transfer will be given by a sum of individual events given by (A2) over all possible initial and final electronic states:

$$
k_{v v^{\prime}}=\frac{2 \pi}{\hbar} \sum_{i} \sum_{k} \lambda_{v v^{\prime}}^{2} \delta\left(E_{i}-E_{k}-E_{v v^{\prime}}\right)
$$

To evaluate the sums in (A3) we will use the concept of density of states $\rho(E)$ defined as the number of states per energy interval such that when multiplied by the probability of occupation of a state given by the Fermi-Dirac distribution function $f(E)$ it gives the number of occupied states in that energy range:

$$
N(E+\mathrm{d} E)=\rho(E) f(E) \mathrm{d} E
$$

The number of terms in the sums of (A3) will be given by the number of occupied states $|i\rangle$ and empty states $|k\rangle$, and thus sums in (A3) can be replaced with integrals as follows:

$$
\begin{aligned}
k_{v v^{\prime}}= & \frac{2 \pi}{\hbar} \int_{0}^{\infty} \mathrm{d} E_{i} \times \int_{0}^{\infty} \mathrm{d} E_{k} \lambda_{v v^{\prime}}^{2} \rho\left(E_{i}\right) \rho\left(E_{k}\right) f\left(E_{i}\right)\left(1-f\left(E_{k}\right)\right) \\
& \times \delta\left(E_{i}-E_{k}-E_{v v^{\prime}}\right)
\end{aligned}
$$

To simplify evaluation of the integrals in (A5) we realize that the coupling matrix element $\lambda_{v v^{\prime}}$ does not depend on energy strongly and can be taken out of the integral. Similarly, we acknowledge that the density of states $\rho(E)$ is a much slower function of energy compared to $f(E)$ and can be taken as constant and moved in front of the integral as well, yielding

$$
k_{v v^{\prime}}=\frac{2 \pi}{h} \lambda_{v v^{\prime}}^{2} \rho_{i} \rho_{k} \int_{0}^{\infty} \mathrm{d} E_{i} \int_{0}^{\infty} \mathrm{d} E_{k} f\left(E_{i}\right)\left(1-f\left(E_{k}\right)\right) \delta\left(E_{i}-E_{k}-E_{v v^{\prime}}\right)
$$

Further on, the delta function reduces the integrals in (A6) to

$$
k_{v v^{\prime}}=\frac{2 \pi}{h} \lambda_{v v^{\prime}}^{2} \rho_{i} \rho_{k} \int_{0}^{\infty} f(E)\left(1-f\left(E-E_{v v^{\prime}}\right)\right) \mathrm{d} E
$$

or

$$
k_{v v^{\prime}}=\alpha_{v v^{\prime}} \int_{0}^{\infty} f(E)\left(1-f\left(E-E_{v v^{\prime}}\right)\right) \mathrm{d} E \text { with } \alpha_{v v^{\prime}} \approx \frac{2 \pi}{h} \lambda_{v v^{\prime}}^{2} \rho^{2}
$$

The integral in (A8) can be taken analytically using the following algebraic equality:

$$
f(E)\left(1-f\left(E-E_{v v^{\prime}}\right)\right)=\frac{f(E)-f\left(E-E_{v v^{\prime}}\right)}{1-e^{E_{v v^{\prime}} / k_{\mathrm{b}} T_{\mathrm{S}}}}
$$


which reduces (A8) to

$$
k_{v v^{\prime}}=\frac{\alpha}{1-e^{E_{v v^{\prime}}} / k_{\mathrm{b}} T_{\mathrm{S}}} \int_{0}^{\infty}\left[f(E)-f\left(E-E_{v v^{\prime}}\right)\right] \mathrm{d} E
$$

The integral in the above can be taken exactly:

$$
\int_{0}^{\infty}\left[f(E)-f\left(E-E_{v v^{\prime}}\right)\right] \mathrm{d} E=k_{\mathrm{b}} T_{\mathrm{S}} \ln \frac{1+e^{-E_{\mathrm{F}} / k_{\mathrm{b}} T_{\mathrm{S}}}}{e^{-E_{\mathrm{F}} / k_{\mathrm{b}} T_{\mathrm{S}}}+e^{E_{\nu \nu^{\prime}} / k_{\mathrm{b}} T_{\mathrm{S}}}}
$$

and can be approximated as

$$
\int_{0}^{\infty}\left[f(E)-f\left(E-E_{v v^{\prime}}\right)\right] \mathrm{d} E \approx-E_{v v^{\prime}}
$$

when

$$
k_{\mathrm{b}} T_{\mathrm{S}} \ll E_{\mathrm{F}}
$$

Substituting (A12) into (A10) we obtain the final expression for the total vibrational energy transfer rate:

$$
k_{v v^{\prime}}=\alpha_{v v^{\prime}} \frac{E_{v v^{\prime}}}{e^{E_{v v^{\prime}} / k_{\mathrm{b}} T}-1}
$$

The condition (A13) establishes the upper temperature limit of the validity of the expression (A14). $\dagger$ For example, in the case of gold $E_{\mathrm{F}} \approx 5.5 \mathrm{eV}$ and the inequality (A13) holds up to $\sim 6 \times 10^{4} \mathrm{~K}$.

\section{Acknowledgements}

This work was supported by the Department of Energy Office of Basic Research under the Grant No. DE-FG02-03ER15441, and by the Partnership for International Research and Education for Electronic Chemistry and Catalysis at Interfaces NSF Grant No. OISE-0530268. I.R. acknowledges support from the Fulbright Foundation. C.B. acknowledges support from the Humboldt foundation.

\section{References}

1 C. T. Rettner, F. Fabre, J. Kimman and D. J. Auerbach, Phys. Rev. Lett., 1985, 55, 1904.

2 N. H. Nahler and A. M. Wodtke, Mol. Phys., 2008, 106, 2227.
3 A. M. Wodtke, D. Matsiev and D. J. Auerbach, Prog. Surf. Sci., 2008, 83, 167.

4 A. M. Wodtke, J. C. Tully and D. J. Auerbach, Int. Rev. Phys. Chem., 2004, 23, 513.

5 D. M. Newns, Surf. Sci., 1986, 171, 600.

6 J. W. Gadzuk and S. Holloway, Phys. Rev. B: Condens. Matter, 1986, 33, 4298.

7 R. Brako and D. M. Newns, Rep. Prog. Phys., 1989, 52, 655.

8 J. C. Tully, J. Chem. Phys., 1990, 93, 1061.

9 A. Gross and W. Brenig, Chem. Phys., 1993, 177, 497.

10 G. A. Gates, G. R. Darling and S. Holloway, J. Chem. Phys., 1994, 101, 6281 .

11 M. Head-Gordon and J. C. Tully, J. Chem. Phys., 1995, 103, 10137.

12 N. Shenvi, S. Roy, P. Parandekar and J. Tully, J. Chem. Phys., 2006, 125, 154703.

13 S. Roy, N. Shenvi and J. C. Tully, J. Phys. Chem. C, 2009, 113, 16311.

14 S. Roy, N. A. Shenvi and J. C. Tully, J. Chem. Phys., 2009, 130, 174716.

15 N. Shenvi, S. Roy and J. C. Tully, J. Chem. Phys., 2009, 130, 174107.

16 N. Shenvi, S. Roy and J. C. Tully, Science, 2009, 326, 829.

17 R. Cooper, I. Rahinov, Z. Li, D. Matsiev, D. J. Auerbach and A. M. Wodtke, Chem. Sci., 2010, 1, 55.

18 Q. Ran, D. Matsiev, D. J. Auerbach and A. M. Wodtke, Phys. Rev. Lett., 2007, 98, 237601.

19 E. K. Watts, J. L. W. Siders and G. O. Sitz, Surf. Sci., 1997, 374, 191.

20 Q. Ran, D. Matsiev, A. M. Wodtke and D. J. Auerbach, Rev. Sci. Instrum., 2007, 78, 104104

21 B. D. Kay, T. D. Raymond and M. E. Coltrin, Phys. Rev. Lett., 1987, 59, 2792.

22 G. J. Schulz, Rev. Mod. Phys., 1973, 45, 423.

23 W. Domcke and L. S. Cederbaum, Phys. Rev. A: At., Mol., Opt. Phys., 1977, 16, 1465.

24 B. N. J. Persson and M. Persson, Solid State Commun., 1980, 36, 175.

25 R. Ryberg, Surf. Sci., 1982, 114, 627.

26 J. W. Gadzuk, Surf. Sci., 1967, 6, 133.

27 D. M. Newns, Phys. Rev., 1969, 178, 1123.

28 J. W. Gadzuk, Surf. Sci., 1974, 43, 44.

29 R. Brako and D. M. Newns, Solid State Commun., 1980, 33, 713.

30 J. W. Gadzuk and H. Metiu, Phys. Rev. B: Condens. Matter, 1980 , 22, 2603.

31 J. W. Gadzuk and J. K. Norskov, J. Chem. Phys., 1984, 81, 2828.

32 P. W. Anderson, Phys. Rev., 1961, 124, 41.

33 D. M. Newns, J. Chem. Phys., 1969, 50, 4572.

34 Y. H. Huang, C. T. Rettner, D. J. Auerbach and A. M. Wodtke, Science, 2000, 290, 111.

35 N. H. Nahler, J. D. White, J. LaRue, D. J. Auerbach and A. M. Wodtke, Science, 2008, 321, 1191. 